\title{
Revelation without presentation: Counterfeit study list yields robust revelation effect
}

\author{
LENORE C. FRIGO, DEBORAH L. REAS, and DENNY C. LECOMPTE \\ Louisiana State University, Baton Rouge, Louisiana
}

\begin{abstract}
This study explores the revelation effect, a recognition memory phenomenon that occurs when test items (or related items) are specially processed before recognition judgment. These revealed items, whether targets or lures, receive a positive response bias. Although the effect occurs across various conditions, it has not been shown to occur when participants make judgments unrelated to episodic memory. We investigated whether the effect would occur when a recognition decision was nominally one of episodic memory, but when a complete episodic event had not occurred. Specifically, participants listened to noise that allegedly masked a list of words (in fact, no words existed). A revelation effect occurred with this pseudo-subliminal procedure, suggesting that the revelation effect need not rely on stimuli recalled through episodic memory but only a specific event to recall. The effect did not occur when participants simply guessed whether words were on an unheard list or made semantic judgments.
\end{abstract}

When test items in recognition tests are somehow disguised or revealed, individuals provide more positive responses for these revealed items than when test items are presented in a normal fashion. This phenomenon, known as the revelation effect, occurs for both target and lure items on recognition tests (Watkins \& Peynircioğlu, 1990). For target items, participants show a greater hit rate for revealed items than for normal items, and similarly, for lures, a greater false alarm rate occurs for revealed items than for normal items.

A variety of procedures generate the revelation effect: It is found when words on a recognition test are revealed letter by letter, presented as anagrams or word fragments to be solved, or shown with individual letters rotated. For example, when participants unscramble the letters ebalt to form the word table and then judge whether table was on the studied list, the participants are more likely to state that table was on the studied list than if it had not been initially presented as an anagram. The revelation effect also occurs when words are presented in a normal fashion on a recognition test but when participants must perform a task on the words before judging whether they were on the study list. For example, participants are more likely to respond positively to a word (e.g., judicial) as having been on the study list if they are required to count the number of ascending letters of another word (e.g., $b$ and $d$ of abandon) than if they simply read the word judicial. Overall, the revelation effect is a robust phenomenon that occurs when words are manipulated in many different ways (see LeCompte, 1995; Luo, 1993; Peynircioğlu \& Tekcan, 1993; Watkins \& Peynircioğlu, 1990; Westerman \& Greene, 1996, 1998). The revelation effect also occurs when num-

Correspondence concerning this article should be addressed to L. C Frigo, College of Southern Idaho, 315 Falls Ave., P.O. Box 1238, Twin Falls, ID 83303 (e-mail: lfrigo@aspen1.csi.cc.id.us). bers rather than words are studied. For example, numbers presented as Roman numerals or as solutions of equations receive more positive responses than do numbers presented in the standard format (Watkins \& Peynircioğlu, 1990). The revelation effect is found both when participants make binary recognition decisions (i.e., yes/no, old/ new) and when they make confidence ratings in regard to these binary decisions. In addition, the revelation effect has been found when participants judge how often an item has occurred in a studied list, with revelation causing participants to judge items as having occurred more frequently (Westerman \& Greene, 1996).

Typically a within-subjects design has been used in revelation effect experiments. However, the effect has also occurred with a between-subjects design. Specifically, Westerman and Greene (1996) found that when a recognition test included either normally presented words or anagrams, participants seeing anagrams gave more positive responses than did those seeing the words in a normal fashion (Westerman \& Greene, 1996).

Strangely, the revelation effect occurs even when the revealed word is not identical to the word to be judged on the recognition test (Westerman \& Greene, 1996). For example, Westerman and Greene (1996) found that when participants solved an anagram for the word raindrop and then immediately judged whether vineyard had been on the studied list, positive responses for vineyard were greater than when it was presented in isolation. Further, the revelation effect occurred for words on a recognition test even when the revelation task involved rearranging unpronounceable strings of letters rather than words (e.g., COEJZWHS; Westerman \& Greene, 1998). Thus, the revelation effect is not limited to situations in which the revelation and to-be-judged words are identical.

Although the revelation effect is quite robust, the phenomenon is not without its limitations. These limitations 
may provide the best clues toward understanding the effect. For example, the revelation effect has not occurred for words when the preceding revelation task has involved numbers rather than words (Westerman \& Greene, 1998). In addition, Watkins and Peynircioğlu (1990) investigated whether the revelation effect would extend to nonepisodic memory tasks. Participants judged words for lexicality, general frequency of occurrence, and typicality as members of categories. For the lexical decision task, participants read low-frequency words (e.g., spiry) and nonwords (e.g., swerth) and judged whether each was an actual word. For the estimation of occurrence of words in everyday life, participants were asked to rate how often they encountered each of a series of words. For the judgment of category typicality, participants were asked to rate how well an exemplar typified a given category. In these three cases, no revelation effect occurred: When words were revealed (by presenting them with individual letters rotated) this manipulation did not yield a positive response bias in contrast to performance with normally presented words. However, a revelation effect did occur for a standard recognition test using the same letter-rotation manipulation. Given these findings, it appears that the revelation effect may be limited to tasks involving an episodic memory event-in particular, a given exposure to a certain list of words or numbers.

Building on Watkins and Peynircioğlu's (1990) finding that the revelation effect does not occur with judgments of words not based on episodic memory, we wanted to test the hypothesis that an episodic memory event is a necessary condition for the revelation effect. That is, the revelation effect might depend on a previous exposure to some particular set of to-be-remembered stimuli. On the other hand, a particular set of events might not be necessary for the revelation effect to occur. Possibly only a suggestion of episodic memory might be necessary. If so, the revelation effect would occur even if there were no to-beremembered items prior to a recognition test.

In order to suggest an event for episodic memory without actually presenting a set of to-be-remembered stimuli, participants in both Experiments 1 and 2 listened to an audio tape purported by the experimenters to contain subliminally presented words. In actuality, there were no words on the tape. A revelation effect occurring in this situation would imply that it does not require an actual episodic memory event to have occurred-that is, an experience of specific stimuli to be remembered at a later time. On the other hand, if the revelation effect failed to occur, we would learn one more boundary of the revelation effect: that it does require actual exposure to a particular set of to-be-remembered stimuli. Our experimental task did not involve a complete episodic memory event in that the participants neither experienced nor remembered any specific events. But we did retain one component of episodic memory — namely, a definite event or episodewhich is said to be relevant to the memory test. Namely, we suggested to the participants that an episode to be recalled occurred at an unconscious level.

\section{EXPERIMENT 1}

\section{Method}

Participants. The participants were 24 undergraduate students at Louisiana State University. They participated in exchange for psychology course extra credit. Four participants were replaced: three failed to complete more than $60 \%$ of anagrams to be solved, and one failed to follow instructions.

Materials. On an audio tape were recorded bursts of AM radio static, each lasting approximately $1 \mathrm{sec}$, with approximately $500 \mathrm{msec}$ between bursts, with a total duration of $90 \mathrm{sec}$. Other than the static noise, no other stimuli were recorded on the audio tape. An answer sheet for a recognition test included 40 seven-letter words (from Gibson \& Watkins, 1988) presented in a fixed, random order on an answer sheet. For each participant, half of these words were presented in a normal format and the other half were presented as anagrams (e.g., SEAORNG for ORANGES). For half of the participants, the odd-numbered items were anagrams, and for the other participants, the even-numbered items were anagrams. The letters of each word were reordered according to a single rule in order to ensure that each anagram would be solved. All words were rearranged by moving the letters in each position of the word into the order 7613245 - for instance, ORANGES would become SEAORNG. After each word, there was a line for participants to write the solution to the anagram and the words yes and no to indicate their response to each word.

Procedure. The participants were tested in small groups of up to 10 individuals. The experimenter explained that the participants would hear an audio tape containing a list of words, but that they would probably not hear the words because they were masked with bursts of static noise. After listening to the tape, the participants were told that they would take a memory test for the words on the tape; if they had not heard any words, they should use their intuition to guess or imagine what words had been on the list. The participants were told to consider each word carefully, even if they felt they were only guessing. The experimenter also explained to the participants how to solve the anagrams. In addition, an example was listed on the answer sheet. The participants solved each anagram, wrote its solution on the answer sheet and circled yes or no, indicating whether they believed that the word had been on the list. For normally presented words, the participants simply circled yes or no. After they had completed the entire task, the participants were debriefed as to the nature of the experiment.

\section{Results}

A typical revelation effect occurred. Participants were more likely to respond positively to revealed items $(M=$ $45.21 \%, S D=23.80$ ) than to normally presented items $(M=31.67 \%, S D=28.78)[t(23)=3.07, p<.01]$. In analyzing recognition test data, researchers typically separate responses to targets and lures. However, in this analysis, we could not make this distinction; no study items had been presented, making all test items lures.

The revelation effect occurred even though there were no true to-be-remembered items before the recognition test. From this result, it appears that a complete episodic memory event is not necessary for the revelation effect to occur. Although participants may have imagined words while listening to the tape, it is improbable that by chance 
participants would have imagined a meaningful proportion of the test words. Furthermore, of participants who provided comments after the experiment, none reported having heard any words on the tape.

\section{EXPERIMENT 2}

In order to verify the results of the unusual procedure of Experiment 1, in Experiment 2 we replicated Experiment 1 , using a different set of stimuli to ensure that the effect would be robust across varying conditions. First, we used a different set of words at test. Second, the words were revealed in a different fashion. Specifically, rather than present them as anagrams, we rotated the revealed words $180^{\circ}$ so that they appeared upside-down. A revelation effect was found with such a manipulation by Watkins and Peynircioglu (1990). We predicted that the revelation effect without actual list presentation would occur even with these variations in the stimuli.

\section{Method}

Participants. Eighty undergraduate students at Louisiana State University volunteered in exchange for psychology course extra credit. Students were tested in their classrooms in groups of approximately 20-30 students.

Materials and Procedure. The audio tape and instructions were identical to those used in Experiment 1 . However, for the recognition test, a different set of words was included. Sixty words were selected from the Toronto Word Pool (Friendly, Franklin, Hoffman, \& Rubin, 1982). Half of the words were randomly assigned to be presented as upside down; the remaining words were presented normally. Two answer sheets were used, so that each word would be presented upside down on one sheet and normally on the other. The participants were instructed to read each word and judge whether it might have been concealed beneath the noise of the audio tape.

\section{Results}

As in Experiment 1, a revelation effect occurred: There were more positive responses for the revealed items $(M=$ $40.3 \%, S D=20.5)$ than for the normally presented items $(M=33.8 \%, S D=18.2)[t(79)=2.76, p<.01]$. Again as in Experiment 1, the revelation effect occurred even when the episodic event to be recalled did not contain stimuli relevant to the recognition test.

\section{EXPERIMENT 3}

The results of both Experiments 1 and 2 suggest that the revelation effect can occur when no items to be remembered are presented but only when a nominal episodic event is suggested. However, one might question whether episodic memory is in any way essential for the revelation effect to occur. The work of Watkins and Peynircioğlu (1990) suggests that episodic memory is essential to the revelation effect, but perhaps their tasks failed to produce the revelation effect for reasons other than the lack of episodic memory. We examined this issue in Experiment 3. This experiment paralleled Experiment 2, but rather than hearing an audio tape, participants were simply asked to imagine that they heard a list of words and to guess whether each test word was on that imagined list. The aim of this experiment was to determine whether the revelation effect without to-be-remembered items could occur even when individuals knew without any doubt that they had not been exposed to any words before the test. In all respects, Experiments 2 and 3 were identical, with the exception that in Experiment 3 participants did not hear words and knew they had not heard any words, whereas in Experiment 2, participants were led to believe that they had been exposed to words but had not actually heard any words either. The results of this experiment should tell us whether the revelation effect was limited to conditions in which there was at least the suggestion of an episodic memory experience.

\section{Method}

Participants. Forty undergraduate students at Louisiana State University volunteered in exchange for psychology course extra credit.

Materials and Procedure. The method was identical to that of Experiment 2. However, instead of their listening to the tape of static noise, the participants were simply told that there was a list of words on an audio tape but that they would not hear this tape. The instructions for the recognition test were identical to those of Experiments 1 and 2, with the exception that the participants were told only to guess whether each item was on the list and were not asked to rely on their memory in any fashion.

\section{Results}

No revelation effect occurred. Revealed items ( $M=$ $48.48 \%, S D=15.57)$ were not chosen at a greater rate than normally presented items $(M=52.72 \%, S D=16.69)$. Although a revelation effect had occurred in Experiments 1 and 2 when participants listened to a tape of noise supposedly embedded with subliminal words, the revelation effect did not occur when participants were asked to simply guess whether words were on an existing list. It appears that the no-memory revelation effect does not occur simply when participants are asked to make guesses about words but only when they are led to believe that some set of stimuli has been presented and may be influencing their memory in some fashion. Thus, the revelation effect seems to require some experience to be called upon, even if it is devoid of to-be-remembered items. Keep in mind, however, that this finding is based on the null hypothesis. It must be admitted that a revelation effect might not have occurred because participants had not followed instructions or attended to individual items when guessing.

\section{EXPERIMENT 4}

To further demonstrate that the revelation effect occurs only under actual experience or at least the suggestion of an episodic memory event, we presented the same set of words within a semantic judgment task rather than a recognition task. In Experiment 4, participants were pre- 
sented with test stimuli identical to those in Experiments 2 and 3 . However, in this experiment, each participant judged whether the words related to him/herself. On the basis of the work of Watkins and Peynircioğlu (1990), who found no revelation effect when participants made semantic judgments about words, it was predicted that no revelation effect should occur.

\section{Method}

Participants. The participants were 32 undergraduate students at Louisiana State University. They participated in exchange for psychology course extra credit.

Materials and Procedure. The method was based on that of Experiments 2 and 3 . The participants were instructed that they would receive a list of words, half of which were printed upside-down. The participants were to decide whether each word related to him- or herself personally and respond accordingly by circling yes or no.

\section{Results}

As predicted, no revelation effect occurred during the semantic judgment task. The participants did not choose revealed items $(M=49.00 \%, S D=18.48)$ at a significantly greater rate than that for normally presented items $(M=48.23 \%, S D=18.44)[t(31)<1]$.

\section{SUMMARY AND CONCLUSIONS}

In Experiments 1 and 2, we tested whether the revelation effect would appear when participants were asked to judge whether they had been exposed to words embedded in static noise, when in fact no words had been presented. In this condition, the revelation effect did occur-that is, participants exhibited a positive response bias for words that were presented in an unusual fashion. In contrast, the revelation effect did not occur when participants simply guessed whether the test items were on a list that they had not heard (Experiment 3 ) or were asked to make a semantic judgment of the same words (Experiment 4). Thus, although the revelation effect does not require that participants actually hear a set of to-be-remembered words, it does seem to require that individuals at least believe that they are making an episodic judgment based on a specific experience. This specific experience need not be truly relevant to the memory test, but individuals must be led to believe that it is.

Seeing that the revelation effect occurs only when there is an episodic memory event, or at least the appearance of one, implies that the effect is somehow related to episodic memory processes. At the same time, this episodic event can be empty of any stimuli directly related to the recognition task. The revelation effect appears to occur as part of the decision-making process associated with a recognition task. One might ask why the revelation effect may be limited to episodic memory and does not extend to semantic memory. A possible explanation is that episodic memory is more vulnerable to disturbance. For instance, the perceived veridicality of episodic memory is based not on actual truth or social consensus but instead on an indi- vidual's personal belief about whether an event has occurred or not (Tulving, 1983). Accordingly, it is possible that, within episodic memory, an individual can easily have the experience of remembering something that has not occurred. This subjective nature of episodic memory may contribute to its vulnerability to a response bias such as the revelation effect. In addition, episodic memory appears to be less robust relative to semantic memory in other fashions (Tulving, 1983): Episodic memory is based on single events rather than repeated exposure, and episodic memory is less well organized and relies more on conscious effort and strategies than does semantic memory, which tends to be more automatic. It may be that the revelation effect comes in at any of these points of vulnerability to alter recollective experience in episodic memory.

Our results are not inconsistent with previously suggested explanations of the revelation effect. However, our results do not provide evidence that supports one over the other. According to Luo (1993), the revelation effect may be the result of an enhanced sense of familiarity for the items that are revealed as compared with normally presented items. This explanation can account for our findings, in that participants would perceive familiarity as relevant to the task of Experiments 1 and 2, where a revelation effect was found, but not relevant to Experiments 3 and 4, where there was no revelation effect. That is, in the first two experiments, participants were exposed to stimuli said to be relevant to the recognition test. Believing that the stimuli had somehow influenced them, the participants would have relied on perceived levels of familiarity for the recognition tests of Experiments 1 and 2 but not during the latter two experiments, in which participants were not exposed to relevant stimuli. The results are equally compatible with the global-matching model of recognition, as applied to the revelation effect by Westerman and Greene (1998). Under the global-matching model, the revelation effect occurs as the result of increased activation in memory after the revelation process. In Experiments 1 and 2, the revelation effect occurred as participants used the increased activation level in their decision-making process on a task that was perceived to involve episodic memory. However, when participants in Experiments 3 and 4 made judgments not related to episodic memory, increased levels of memory activation played no role, and thus a revelation effect did not occur.

\section{REFERENCES}

Friendly, M., Franklin, P. E., Hoffman, D., \& Rubin, D. C. (1982). The Toronto Word Pool: Norms for imagery, concreteness, orthographic variables, and grammatical usage for 1,080 words. Behavior Methods \& Instrumentation, 14, 375-399.

Gibson, J. M., \& Watkins, M. J. (1988). A pool of 1,086 words with unique two-letter fragments. Behavior Research Methods, Instruments, \& Computers, 20, 390-397.

LECOMPTE, D. C. (1995). Recollective experience in the revelation effect: Separating the contributions of recollection and familiarity. Memory \& Cognition, 23, 324-334.

Luo, C. R. (1993). Enhanced feeling of recognition: Effects of identify- 
ing and manipulating test items on recognition memory. Journal of Experimental Psychology: Learning, Memory, \& Cognition, 19, $405-$ 413.

Peynircioǧlu, Z. F., \& Tekcan, A. İ. (1993). Revelation effect: Effort or priming does not create the sense of familiarity. Journal of Experimental Psychology: Learning, Memory, \& Cognition, 19, 382-388. Tulving, E. (1983). Elements of episodic memory. New York: Oxford University Press.

Watkins, M. J., \& PeynircioğLu, Z. F. (1990). The revelation effect: When disguising test items induces recognition. Journal of Experimental Psychology: Learning, Memory, \& Cognition, 16, 1012-1020.
Westerman, D. L., \& Greene, R. L. (1996). On the generality of the revelation effect on memory. Journal of Experimental Psychology: Learning, Memory, \& Cognition, 22, 1147-1153.

Westerman, D. L., \& GreENE, R. L. (1998). The revelation that the revelation effect is not due to revelation. Journal of Experimental Psychology: Learning, Memory, \& Cognition, 24, 377-386.

(Manuscript received December 4, 1997; revision accepted for publication April 6, 1998.) 